\title{
Generalized Finsler-Hadwiger type inequalities for simplices and applications
}

Shiguo Yang ${ }^{1,2}$ and Wen Wang ${ }^{1 *}$

"Correspondence: wenwang1985@163.com

'School of Mathematics and Statistics, Hefei Normal University, Hefei, Anhui 230601, P.R. China Full list of author information is available at the end of the article

\begin{abstract}
For an $n$-dimensional simplex in the Euclidean space $E^{n}$, we establish some generalized Finsler-Hadwiger inequalities. By using these inequalities, generalizations of some well-known important inequalities for simplices are obtained.
\end{abstract}

MSC: $51 \mathrm{M} 20 ; 52 \mathrm{~A} 40$

Keywords: simplex; volume; circumradius; inradius; inequality

\section{Introduction}

Let $A B C$ be a triangle (in the Euclidean plane) of area $S$ and sides $a, b, c$. The following inequality $[1,2]$ is well known:

$$
a^{2}+b^{2}+c^{2} \geq 4 \sqrt{3} S+(a-b)^{2}+(b-c)^{2}+(c-a)^{2} .
$$

Equality holds iff this triangle is regular.

Let $\Omega_{n}$ be an $n$-dimensional simplex (in the $n$-dimensional Euclidean space $E^{n}$ ) of volume $V$ and edge lengths $a_{i}\left(i=1,2, \ldots, \frac{1}{2} n(n+1)\right)$. Let $A_{i}(i=0,1, \ldots, n)$ be the vertices of $\Omega_{n}, F_{i}$ be the (n-1)-dimensional volume of the $i$ th face $f_{i}=A_{0} \cdots A_{i-1} A_{i+1} \cdots A_{n+1}((n-1)$ dimensional simplex) for $i=0,1, \ldots, n$. In 1989, Chen and Ma extended the inequality (1.1) to an $n$-simplex and established Finsler-Hadwiger type inequality for the edge length and the volume of an $n$-simplex as follows [3, 4]:

$$
\sum_{i=1}^{\frac{1}{2} n(n+1)} a_{i}^{2} \geq n(n+1)\left[\frac{(n !)^{2}}{n+1}\right]^{\frac{1}{n}} V^{\frac{2}{n}}+\frac{2}{n(n-1)} \sum_{1 \leq i<j \leq \frac{1}{2} n(n+1)}\left(a_{i}-a_{j}\right)^{2} .
$$

Equality holds iff $\Omega_{n}$ is regular.

In 1997, Leng and Tang established another kind of Finsler-Hadwiger type inequality for the face areas and the volume of an $n$-simplex as follows $[4,5]$ :

$$
\sum_{i=0}^{n} F_{i}^{2} \geq \frac{n^{3}(n+1)^{\frac{1}{n}}}{(n !)^{\frac{2}{n}}} V^{\frac{2(n-1)}{n}}+\frac{1}{n-1} \sum_{0 \leq i<j \leq n}\left(F_{i}-F_{j}\right)^{2} .
$$

Equality holds iff $\Omega_{n}$ is regular.

In this paper, we will discuss problem for generalized Finsler-Hadwiger type inequalities for $k$-dimensional face areas and the volume of an $n$-simplex, and establish generalized

\section{楚 Springer}

(C) 2015 Yang and Wang; licensee Springer. This is an Open Access article distributed under the terms of the Creative Commons Attribution License (http://creativecommons.org/licenses/by/4.0), which permits unrestricted use, distribution, and reproduction in any medium, provided the original work is properly credited. 
Finsler-Hadwiger type inequalities for an $n$-simplex. From these inequalities, generalizations of some inequalities for an $n$-simplex are obtained.

\section{Main results}

Let $R$ and $r$ be the circumradius and the inradius of the simplex $\Omega_{n}$, respectively. Let $V_{0}$ denote the volume of the $n$-dimensional regular simplex with circumradius $R$. For $k=0,1, \ldots, n-1$, we put $\mu_{n, k}=\left(\begin{array}{c}n+1 \\ k+1\end{array}\right)=\frac{(n+1) !}{(k+1) !(n-k) !}$. Let $V_{i}(k)\left(i=1,2, \ldots, \mu_{n, k}\right)$ denote the $k$-dimensional volumes of the $k$-dimensional subsimplices of $\Omega_{n}$. Our main results are the following theorems.

Theorem 1 Let $\Omega_{n}$ be an $n$-simplex, nature number $k \in[1, n-1]$, real numbers $\alpha \in(0,1]$ and $\lambda \in(0, n+1-k]$. Then we have

$$
\begin{aligned}
\sum_{i=1}^{\mu_{n, k}} V_{i}^{2 \alpha}(k) \geq & \left(\frac{R}{n r}\right)^{\frac{2 k \alpha}{n(n+1)(n-1)^{2}}} \cdot \mu_{n, k} \cdot\left[\frac{k+1}{(k !)^{2}}\left(\frac{(n !)^{2}}{n+1}\right)^{\frac{k}{n}}\right]^{\alpha} V^{\frac{2 k \alpha}{n}} \\
& +\frac{1}{\mu_{n, k}-\lambda} \sum_{1 \leq i<j \leq \mu_{n, k}}\left(V_{i}^{\alpha}(k)-V_{j}^{\alpha}(k)\right)^{2} .
\end{aligned}
$$

Equality holds iff $\Omega_{n}$ is regular.

Theorem 2 Let $\Omega_{n}$ be an n-simplex, nature number $k \in[1, n-1]$, real numbers $\alpha \in(0,1]$ and $\lambda \in(0, n+1-k]$. Then we have

$$
\begin{aligned}
\sum_{i=1}^{\mu_{n, k}} V_{i}^{2 \alpha}(k) \geq & \left(\frac{V_{0}}{V}\right)^{\frac{2 k \alpha}{n(n+1)(n-1)^{2}}} \cdot \mu_{n, k} \cdot\left[\frac{k+1}{(k !)^{2}}\left(\frac{(n !)^{2}}{n+1}\right)^{\frac{k}{n}}\right]^{\alpha} V^{\frac{2 k \alpha}{n}} \\
& +\frac{1}{\mu_{n, k}-\lambda} \sum_{1 \leq i<j \leq \mu_{n, k}}\left(V_{i}^{\alpha}(k)-V_{j}^{\alpha}(k)\right)^{2} .
\end{aligned}
$$

Equality holds iff $\Omega_{n}$ is regular.

Notice From the inequality $R \geq n r$ and Lemma 3, we have $\frac{R}{n r} \geq 1$ and $\frac{V_{0}}{V} \geq 1$, respectively. Further, according to the conditions of equalities hold, we now consider a class of $n$-simplices $\varphi$ inscribed in an $(n-1)$-dimensional sphere of radius $R$ (where $R$ is constant number). If the radius $r$ of an $n$-simplex in $\varphi$ is sufficiently small, then $\frac{R}{n r}$ is large enough. Similarly, if the volume $V$ of an $n$-simplex in $\varphi$ is sufficiently small, then $\frac{V_{0}}{V}$ is sufficiently large.

Take $\lambda=n+1-k$, from Theorem 1 or Theorem 2 we derive an inequality for a simplex as follows.

Corollary 1 Let $\Omega_{n}$ be an $n$-simplex and real number $\alpha \in(0,1]$. Then

$$
\begin{aligned}
\sum_{i=1}^{\mu_{n, k}} V_{i}^{2 \alpha}(k) \geq & \mu_{n, k} \cdot\left[\frac{k+1}{(k !)^{2}}\left(\frac{(n !)^{2}}{n+1}\right)^{\frac{k}{n}}\right]^{\alpha} V^{\frac{2 k \alpha}{n}} \\
& +\frac{1}{\mu_{n, k}-(n+1-k)} \sum_{1 \leq i<j \leq \mu_{n, k}}\left(V_{i}^{\alpha}(k)-V_{j}^{\alpha}(k)\right)^{2} .
\end{aligned}
$$

Equality holds iff $\Omega_{n}$ is regular. 
By taking $k=1$ and $\alpha=1$ in the inequality (2.3) we can obtain the inequality (1.2). Take $k=n-1$ and $\alpha=1$ in the inequality (2.3) we can obtain the inequality (1.3).

Take $k=1, \alpha=1$ and $\lambda=n$ in Theorem 1 and Theorem 2 , we get generalizations of the inequality (1.2) as follows.

Corollary 2 For an $n$-simplex $\Omega_{n}$, we have

$$
\begin{aligned}
\sum_{i=1}^{\frac{1}{2} n(n+1)} a_{i}^{2} \geq & \left(\frac{R}{n r}\right)^{\frac{2}{n(n+1)(n-1)^{2}}} \cdot n(n+1) \cdot\left[\frac{(n !)^{2}}{n+1}\right]^{\frac{1}{n}} V^{\frac{2}{n}} \\
& +\frac{2}{n(n-1)} \sum_{1 \leq i<j \leq \frac{1}{2} n(n+1)}\left(a_{i}-a_{j}\right)^{2}, \\
\sum_{i=1}^{\frac{1}{2} n(n+1)} a_{i}^{2} \geq & \left(\frac{V_{0}}{V}\right)^{\frac{2}{n(n+1)(n-1)^{2}}} \cdot n(n+1) \cdot\left[\frac{(n !)^{2}}{n+1}\right]^{\frac{1}{n}} V^{\frac{2}{n}} \\
& +\frac{2}{n(n-1)} \sum_{1 \leq i<j \leq \frac{1}{2} n(n+1)}\left(a_{i}-a_{j}\right)^{2} .
\end{aligned}
$$

Equality holds iff $\Omega_{n}$ is regular.

Take $k=n-1, \alpha=1$, and $\lambda=2$ in Theorem 1 and Theorem 2; we get generalizations of the inequality (1.3) as follows.

Corollary 3 Let $\Omega_{n}$ be an n-simplex. Then we have

$$
\begin{aligned}
& \sum_{i=0}^{n} F_{i}^{2} \geq\left(\frac{R}{n r}\right)^{\frac{2}{n\left(n^{2}-1\right)}} \cdot \frac{n^{3}(n+1)^{\frac{1}{n}}}{(n !)^{\frac{2}{n}}} V^{\frac{2(n-1)}{n}}+\frac{1}{n-1} \sum_{0 \leq i<j \leq n}\left(F_{i}-F_{j}\right)^{2}, \\
& \sum_{i=0}^{n} F_{i}^{2} \geq\left(\frac{V_{0}}{V}\right)^{\frac{2}{n\left(n^{2}-1\right)}} \cdot \frac{n^{3}(n+1)^{\frac{1}{n}}}{(n !)^{\frac{2}{n}}} V^{\frac{2(n-1)}{n}}+\frac{1}{n-1} \sum_{0 \leq i<j \leq n}\left(F_{i}-F_{j}\right)^{2} .
\end{aligned}
$$

Equality holds iff $\Omega_{n}$ is regular.

To prove the above theorems, we need some lemmas as follows.

Lemma $1[4,6]$ For an n-simplex $\Omega_{n}$, we have

$$
\left(\prod_{i=0}^{n} F_{i}\right)^{n-1} \geq\left[\frac{n^{3(n-1)}}{2(n+1)^{n-2}(n !)^{2}}\right]^{\frac{n+1}{2}} V^{n^{2}-n-2}\left(\prod_{i=1}^{\frac{1}{2} n(n+1)} a_{i}\right)^{\frac{2}{n}}
$$

with equality iff $\Omega_{n}$ is regular.

Lemma $2[4,7]$ Let $\Omega_{n}$ be an n-simplex with edge lengths $a_{i j}=\left|A_{i} A_{j}\right|(0 \leq i<j \leq n)$, then we have

$$
\prod_{0 \leq i<j \leq n} a_{i j} \geq\left(\frac{2^{n+1}}{n}\right)^{\frac{n}{4}}(n !)^{\frac{n}{2}}(V R)^{\frac{n}{2}}
$$

with equality iff $\Omega_{n}$ is regular. 
Lemma $3[4,8]$ For an n-simplex $\Omega_{n}$, we have

$$
V \leq\left[\frac{(n+1)^{n+1}}{(n !)^{2} n^{n}}\right]^{\frac{1}{2}} R^{n}
$$

with equality iff $\Omega_{n}$ is regular.

Lemma $4[4,6]$ For an n-simplex $\Omega_{n}$, we have

$$
\left(\prod_{i=0}^{n} F_{i}\right)^{n-1} \geq\left[n^{3 n^{2}-4}(n+1)^{-(n+1)(n-2)}\right]^{\frac{1}{2}}(n !)^{-n} V^{n^{2}-n-1} R .
$$

Equality holds iff $\Omega_{n}$ is regular.

Lemma $5[4,9]$ Let $\Omega_{n}$ be an n-simplex and natural number $k \in[1, n-1)$. Then

$$
\frac{k !}{\sqrt{k+1}}\left(\prod_{i=1}^{\mu_{n, k}} V_{i}(k)\right)^{\frac{1}{\mu_{n, k}}} \geq\left[\frac{(n-1) !}{\sqrt{n}}\left(\prod_{i=0}^{n} F_{i}\right)^{\frac{1}{n+1}}\right]^{\frac{k}{n-1}},
$$

with equality iff $\Omega_{n}$ is regular.

Lemma 6 For an n-simplex $\Omega_{n}$, we have

$$
V \geq \frac{(n+1)^{\frac{n+1}{2}} n^{\frac{n^{2}-1}{2 n}}}{n !} r^{\frac{n^{2}-1}{n}} R^{\frac{1}{n}}
$$

with equality iff $\Omega_{n}$ is regular.

Proof Let $P$ be an interior point of $\Omega_{n}$ and $d_{i}$ the distance from the point $P$ to the $i$ th face $f_{i}$ of $\Omega_{n}$ for $i=0,1, \ldots, n$. Obviously, we have $\sum_{i=0}^{n} d_{i} F_{i}=n V$. Then

$$
\sum_{i=0}^{n} \frac{d_{i} F_{i}}{n V}=1
$$

Using the arithmetic-geometric means inequality we get

$$
\prod_{i=0}^{n} \frac{d_{i} F_{i}}{n V} \leq\left(\frac{1}{n+1} \sum_{i=0}^{n} \frac{d_{i} F_{i}}{n V}\right)^{n+1}=\frac{1}{(n+1)^{n+1}},
$$

namely

$$
\left(\prod_{i=0}^{n} d_{i}\right)^{n-1} \leq \frac{(n V)^{n^{2}-1}}{(n+1)^{n^{2}-1}\left(\prod_{i=0}^{n} F_{i}\right)^{n-1}} .
$$

Substituting (2.11) into (2.14) we get

$$
\left(\prod_{i=0}^{n} d_{i}\right)^{n-1} \leq(n !)^{n}\left[(n+1)^{n(n+1)} n^{n^{2}-2}\right]^{-\frac{1}{2}} \frac{V^{n}}{R} .
$$


We now take the point $P$ is the incenter of $\Omega_{n}$, then $d_{i}=r$ for $i=0,1, \ldots, n$. Thus from (2.15) we can obtain the inequality (2.13). It is easy to see that equality holds in (2.13) iff $\Omega_{n}$ is regular.

Lemma 7 For an $n$-simplex $\Omega_{n}$ and $k=1,2, \ldots, n-1$, we have

$$
\begin{aligned}
& \left(\prod_{i=1}^{\mu_{n, k}} V_{i}(k)\right)^{\frac{1}{\mu_{n, k}}} \geq\left(\frac{R}{n r}\right)^{\frac{k}{n(n+1)(n-1)^{2}}} \frac{\sqrt{k+1}}{k !}\left(\frac{n !}{\sqrt{n+1}} V\right)^{\frac{k}{n}}, \\
& \left(\prod_{i=1}^{\mu_{n, k}} V_{i}(k)\right)^{\frac{1}{\mu_{n, k}}} \geq\left(\frac{V_{0}}{V}\right)^{\frac{k}{n(n+1)(n-1)^{2}}} \frac{\sqrt{k+1}}{k !}\left(\frac{n !}{\sqrt{n+1}} V\right)^{\frac{k}{n}} .
\end{aligned}
$$

Equality holds iff $\Omega_{n}$ is regular.

Proof We have the well-known inequality $[4,10,11]$

$$
V \leq \frac{(n+1)^{\frac{n+1}{2}}}{n^{\frac{n-2}{2}} n !} R^{n-1} r
$$

Equality holds iff $\Omega_{n}$ is regular.

Using (2.18) and (2.11) we get

$$
\left(\prod_{i=0}^{n} F_{i}\right)^{n-1} \geq\left[\frac{n^{3 n^{2}-4}}{(n+1)^{n^{2}-n-2}}\right]^{\frac{1}{2}} \frac{V^{\frac{\left(n^{2}-1\right)(n-1)}{n}} R}{\left(n !^{n}\right) V^{\frac{1}{n}}} .
$$

From (2.19) and (2.18) we have

$$
\left(\prod_{i=0}^{n} F_{i}\right)^{\frac{1}{n+1}} \geq\left(\frac{R}{n r}\right)^{\frac{1}{n\left(n^{2}-1\right)}} \frac{n^{\frac{3}{2}}}{(n !)^{\frac{1}{n}}(n+1)^{\frac{n-1}{2 n}}} V^{\frac{n-1}{n}} .
$$

Thus, inequality (2.16) is valid for $k=n-1$. We now prove that inequality (2.16) is valid for $1 \leq k<n-1$. Substituting (2.20) into (2.12) we can obtain inequality (2.16). So the inequality (2.16) is valid for $1 \leq k<n-1$. It is easy to see that equality holds in (2.16) iff $\Omega_{n}$ is regular.

By Lemma 3 we have

$$
V_{0}=\left[\frac{(n+1)^{n+1}}{(n !)^{2} n^{n}}\right]^{\frac{1}{2}} R^{n}
$$

Substituting (2.21) into the right of (2.9) we get

$$
\prod_{i=1}^{\frac{1}{2} n(n+1)} a_{i} \geq\left(\frac{V_{0}}{V}\right)^{\frac{1}{2}}\left(\frac{2^{n}}{n+1}\right)^{\frac{n+1}{4}}(n ! V)^{\frac{n+1}{2}} .
$$

From (2.8) and (2.22) we have

$$
\left(\prod_{i=0}^{n} F_{i}\right)^{\frac{1}{n+1}} \geq\left(\frac{V_{0}}{V}\right)^{\frac{1}{n\left(n^{2}-1\right)}} \frac{n^{\frac{3}{2}}}{(n !)^{\frac{1}{n}}(n+1)^{\frac{n-1}{2 n}}} V^{\frac{n-1}{n}} .
$$


Thus the equality (2.17) is valid for $k=n-1$. Now we prove that inequality (2.17) is valid for $1 \leq k<n-1$. Substituting (2.23) into the right of (2.12) we can obtain inequality (2.17). So the inequality (2.17) is valid for $1 \leq k<n-1$. It is easy to see that equality holds in (2.16) iff $\Omega_{n}$ is regular.

Lemma $8[4,5]$ Let $\Omega_{n}$ be an n-simplex and real number $\alpha \in(0,1]$. Then

$$
2 \sum_{0 \leq i<j \leq n} F_{i}^{\alpha} F_{j}^{\alpha}-\sum_{i=0}^{n} F_{i}^{2 \alpha} \geq\left(n^{2}-1\right)\left[\frac{n^{3}}{n+1}\left(\frac{n+1}{(n !)^{2}}\right)^{\frac{1}{n}}\right]^{\alpha} V^{\frac{2(n-1) \alpha}{n}},
$$

with equality iff $\Omega_{n}$ is regular.

Proof of Theorem 1 and Theorem 2 The inequality (2.1) can be written as

$$
\begin{aligned}
& \sum_{i=1}^{\mu_{n, k}} V_{i}^{2 \alpha}(k)+2 \sum_{1 \leq i<j \leq \mu_{n, k}} V_{i}^{\alpha}(k) V_{j}^{\alpha}(k)-\lambda \sum_{i=1}^{\mu_{n, k}} V_{i}^{2 \alpha}(k) \\
& \geq\left(\frac{R}{n r}\right)^{\frac{2 k \alpha}{n(n+1)(n-1)^{2}}} \cdot \mu_{n, k}\left(\mu_{n, k}-\lambda\right) \cdot\left[\frac{\sqrt{k+1}}{k !}\left(\frac{n !}{\sqrt{n+1}}\right)^{\frac{k}{n}}\right]^{2 \alpha} V^{\frac{2 k \alpha}{n}} .
\end{aligned}
$$

Now we prove that the inequality $(2.25)$ is valid.

Let $p(k, \alpha, \delta)$ denote the left of the inequality (2.25). Then

$$
\begin{aligned}
p(k, \alpha, \delta)= & 2 \sum_{1 \leq i<j \leq \mu_{n, k}} V_{i}^{\alpha}(k) V_{j}^{\alpha}(k)-(n-k) \sum_{i=1}^{\mu_{n, k}} V_{i}^{2 \alpha}(k)+(n+1-k-\lambda) \sum_{i=1}^{\mu_{n, k}} V_{i}^{2 \alpha}(k) \\
= & \sum_{\left(i_{1}, i_{2}, \ldots, i_{k+2}\right) \in \sigma}\left(2 \sum_{1 \leq p<q \leq k+2} V_{i_{p}}^{\alpha}(k) V_{i_{q}}^{\alpha}(k)-\sum_{p=1}^{k+2} V_{i_{p}}^{2 \alpha}(k)\right) \\
& +2 \sum_{i_{p}, i_{q} \in \tau} V_{i_{p}}^{\alpha}(k) V_{i_{q}}^{\alpha}(k)+(n+1-k-\lambda) \sum_{i=1}^{\mu_{n, k}} V_{i}^{2 \alpha}(k),
\end{aligned}
$$

where $\sigma=\left\{\left(i_{1}, i_{2}, \ldots, i_{k+2}\right) \mid\right.$ there exists a $(k+1)$-subsimplex $A_{i_{1}} A_{i_{2}} \cdots A_{i_{k+2}}$ of $\Omega_{n}$, such that its $k+2$ side areas are $\left.V_{i_{1}}(k), V_{i_{2}}(k), \ldots, V_{i_{k+2}}(k)\left(1 \leq i_{1}<i_{2}<\cdots<i_{k+2} \mu_{n, k}\right)\right\}$, and $\tau=\left\{\left(i_{p}, i_{q}\right) \mid\right.$ there is not a $(k+1)$-subsimplex of $\Omega_{n}$, such that its two side areas are $\left.V_{i_{p}}(k), V_{i_{q}}(k)\right\}$.

On the other hand, we easily get

$$
|\sigma|=\left(\begin{array}{l}
n+1 \\
k+2
\end{array}\right), \quad|\tau|=\left(\begin{array}{c}
\mu_{n, k} \\
2
\end{array}\right)-\left(\begin{array}{c}
n+1 \\
k+2
\end{array}\right)=\frac{1}{2}\left[\mu_{n, k}-(n-k)(k+1)-1\right] .
$$

We put $m=|\sigma|$, then $m=\left(\begin{array}{c}n+1 \\ k+2\end{array}\right)=\frac{n-k}{k+2} \cdot \mu_{n, k}$. If $\left(i_{1}, i_{2}, \ldots, i_{k+2}\right) \in \sigma$, we use $V_{i}(k+1)$ to denote the volume of $k+1$-subsimplex with side areas $V_{i_{1}}(k), V_{i_{2}}(k), \ldots, V_{i_{k+2}}(k)$. By Lemma 8 we have

$$
\begin{aligned}
& 2 \sum_{1 \leq p<q \leq k+2} V_{i_{p}}^{\alpha}(k) V_{i_{q}}^{\alpha}(k)-\sum_{p=1}^{k+2} V_{i_{p}}^{2 \alpha}(k) \\
& \quad \geq k(k+2)\left[\frac{(k+1)^{3}}{k+2}\left(\frac{k+2}{(k+1) !^{2}}\right)^{\frac{1}{k+1}}\right]^{\alpha}\left(V_{i}(k+1)\right)^{\frac{2 k \alpha}{k+1}} .
\end{aligned}
$$

Equality holds iff the simplex $A_{i_{1}} A_{i_{2}} \cdots A_{i_{k+2}}$ is regular. 
Using the arithmetic-geometric means inequality, (2.27), and (2.16) we get

$$
\begin{aligned}
I_{1} & =\sum_{\left(i_{1}, i_{2}, \ldots, i_{k+2}\right) \in \sigma}\left(2 \sum_{1 \leq p<q \leq k+2} V_{i_{p}}^{\alpha}(k) V_{i_{q}}^{\alpha}(k)-\sum_{p=1}^{k+2} V_{i_{p}}^{2 \alpha}(k)\right) \\
& \geq \sum_{\left(i_{1}, 1_{2}, \ldots, i_{k+2}\right) \in \sigma} k(k+2)\left[\frac{(k+1)^{3}}{k+2}\left(\frac{k+2}{(k+1) !^{2}}\right)^{\frac{1}{k+1}}\right]^{\alpha}\left(V_{i}(k+1)\right)^{\frac{2 k \alpha}{k+1}} \\
& \geq \mu_{n, k+1} k(k+2)\left[\frac{(k+1)^{3}}{k+2}\left(\frac{k+2}{(k+1) !^{2}}\right)^{\frac{1}{k+1}}\right]^{\alpha}\left(\prod_{i=1}^{\mu_{n, k+1}} V_{i}(k+1)\right)^{\frac{2 k \alpha}{(k+1) \mu_{n, k+1}}} \\
& \geq\left(\frac{R}{n r}\right)^{\frac{2 k \alpha}{n(n+1)(n-1)^{2}}} k(k+2)\left[\frac{(k+1)^{3}}{k+2}\left(\frac{k+2}{(k+1) !^{2}}\right)^{\frac{1}{k+1}}\right]^{\alpha} \\
& \cdot \mu_{n, k+1}\left[\frac{\sqrt{k+2}}{(k+1) !}\left(\frac{n !}{\sqrt{n+1}}\right)^{\frac{k+1}{n}}\right]^{\frac{2 k \alpha}{k+1}} V^{\frac{2 k \alpha}{n}} \\
& =\left(\frac{R}{n r}\right)^{\frac{2 k \alpha}{n(n+1)(n-1)^{2}}} \mu_{n, k+1} k(n-k)\left[\frac{\sqrt{k+1}}{(k) !}\left(\frac{n !}{\sqrt{n+1}}\right)^{\frac{k}{n}}\right]^{2 \alpha} V^{\frac{2 k \alpha}{n}} .
\end{aligned}
$$

By the arithmetic-geometric means inequality, and by (2.16), we get

$$
\begin{aligned}
I_{2} & =\sum_{\left(i_{p}, i_{q}\right) \in \tau} 2 V_{i_{p}}^{\alpha}(k) V_{i_{q}}^{\alpha}(k)+(n+1-k-\lambda) \sum_{i=1}^{\mu_{n, k}} V_{i}^{2 \alpha}(k) \\
& \geq \mu_{n, k}\left[\mu_{n, k}-(n-k)(k+1)-1\right]\left(\prod_{i=1}^{\mu_{n, k}} V_{i}(k)\right)^{\frac{2 \alpha}{\mu_{n, k}}}+(n+1-k-\lambda) \mu_{n, k}\left(\prod_{i=1}^{\mu_{n, k}} V_{i}(k)\right)^{\frac{2 \alpha}{\mu_{n, k}}} \\
& \geq\left(\frac{R}{n r}\right)^{\frac{2 k \alpha}{n(n+1)(n-1)^{2}}} \mu_{n, k}\left[\mu_{n, k}-(n-k) k-\lambda\right]\left[\frac{\sqrt{k+1}}{(k) !}\left(\frac{n !}{\sqrt{n+1}}\right)^{\frac{k}{n}}\right]^{2 \alpha} V^{\frac{2 k \alpha}{n}} \cdot
\end{aligned}
$$

From (2.26), (2.28), and (2.29) we get

$$
\begin{aligned}
p(k, \alpha, \lambda) & =I_{1}+I_{2} \\
& \geq\left(\frac{R}{n r}\right)^{\frac{2 k \alpha}{n(n+1)(n-1)^{2}}} \mu_{n, k}\left[\mu_{n, k}-\lambda\right]\left[\frac{\sqrt{k+1}}{(k) !}\left(\frac{n !}{\sqrt{n+1}}\right)^{\frac{k}{n}}\right]^{2 \alpha} V^{\frac{2 k \alpha}{n}} .
\end{aligned}
$$

Thus the equality (2.25) is valid. It is easy to prove that the equality holds iff $\Omega_{n}$ is regular.

Similarly, we can prove that (2.2) is true by the same method.

\section{Some applications}

Geometric inequalities for simplices which are the simplest and useful polytopes have been a very attractive subject for a long time. Mitrinović, Pečarić, Volenec, Zhang and Yang and other authors have obtained a great number of elegant results (see [8]). Specially, we mention the well-known Euler inequality for a simplex, inequalities for the width of a simplex, and inequalities related the interior point of a simplex. In this section we shall improve these inequalities by using the results in the above section. 
LF Tóth extended the Euler inequality $R \geq 2 r$ for a triangle to an $n$-dimensional simplex and obtained the Euler inequality for an $n$-simplex as follows $[8,10]$ :

$$
R \geq n r
$$

Equality holds iff $\Omega_{n}$ is regular.

Let $O$ and $I$ denote the circumcenter and the incenter of an $n$-simplex $\Omega_{n}$, respectively. Let $G$ be the barycenter of $\Omega_{n}$. Klamkin $[8,12]$ and Yang and Wang obtained the following generalizations of (3.1):

$$
\begin{aligned}
& R^{2}-|O I|^{2} \geq(n r)^{2}, \\
& R^{2}-|O G|^{2} \geq(n r)^{2} .
\end{aligned}
$$

Equality holds iff $\Omega_{n}$ is regular.

We shall use these results in the above section to study related inequalities, and we obtain a strengthened Euler inequality as follows.

Theorem 3 For an $n$-simplex $\Omega_{n}$ and $k=1,2, \ldots, n-1$, we have

$$
\begin{aligned}
& \left(R^{2}-|O G|^{2}\right)^{k} \geq\left(\frac{R}{n r}\right)^{\frac{2 k}{n(n+1)(n-1)^{2}}}(n r)^{2 k}+\frac{1}{\mu_{n, k}-(n+1-k)} \sum_{1 \leq i<j \leq \mu_{n, k}}\left(V_{i}(k)-V_{j}(k)\right)^{2} \\
& \left(R^{2}-|O G|^{2}\right)^{k} \geq\left(\frac{V_{0}}{V}\right)^{\frac{2 k}{n(n+1)(n-1)^{2}}}(n r)^{2 k}+\frac{1}{\mu_{n, k}-(n+1-k)} \sum_{1 \leq i<j \leq \mu_{n, k}}\left(V_{i}(k)-V_{j}(k)\right)^{2} .
\end{aligned}
$$

Equality holds iff $\Omega_{n}$ is regular.

If take $k=1$ and $k=n-1$ in (3.4) and (3.5), we get two corollaries as follows.

Corollary 4 For an $n$-simplex $\Omega_{n}$ and $k=1,2, \ldots, n-1$, we have

$$
\begin{aligned}
& R^{2}-|O G|^{2} \geq\left(\frac{R}{n r}\right)^{\frac{2}{n(n+1)(n-1)^{2}}}(n r)^{2}+\frac{2}{n(n-1)} \sum_{1 \leq i<j \leq \frac{1}{2} n(n+1)}\left(a_{i}-a_{j}\right)^{2}, \\
& R^{2}-|O G|^{2} \geq\left(\frac{V_{0}}{V}\right)^{\frac{2}{n(n+1)(n-1)^{2}}}(n r)^{2}+\frac{2}{n(n-1)} \sum_{1 \leq i<j \leq \frac{1}{2} n(n+1)}\left(a_{i}-a_{j}\right)^{2},
\end{aligned}
$$

with equality iff $\Omega_{n}$ is regular.

Corollary 5 For an n-simplex $\Omega_{n}$ and $k=1,2, \ldots, n-1$, we have

$$
\left(R^{2}-|O G|^{2}\right)^{n-1} \geq\left(\frac{R}{n r}\right)^{\frac{2}{n\left(n^{2}-1\right)}}(n r)^{2(n-1)}+\frac{1}{n-1} \sum_{0 \leq i<j \leq n}\left(F_{i}-F_{j}\right)^{2},
$$




$$
\left(R^{2}-|O G|^{2}\right)^{n-1} \geq\left(\frac{V_{0}}{V}\right)^{\frac{2}{n\left(n^{2}-1\right)}}(n r)^{2(n-1)}+\frac{1}{n-1} \sum_{0 \leq i<j \leq n}\left(F_{i}-F_{j}\right)^{2} .
$$

Equality holds iff $\Omega_{n}$ is regular.

Proof of Theorem 3 If take $\alpha=1$ and $\lambda=n+1-k$ in (2.1) and (2.2), we obtain

$$
\begin{aligned}
\sum_{i=1}^{\mu_{n, k}} V_{i}^{2}(k) \geq & \left(\frac{R}{n r}\right)^{\frac{2}{n(n+1)(n-1)^{2}}} \mu_{n, k} \cdot \frac{k+1}{(k !)^{2}}\left(\frac{(n !)^{2}}{n+1}\right)^{\frac{k}{n}} V^{\frac{2 k}{n}} \\
& +\frac{1}{\mu_{n, k}-(n+1-k)} \sum_{1 \leq i<j \leq \mu_{n, k}}\left(V_{i}(k)-V_{j}(k)\right)^{2}, \\
\sum_{i=1}^{\mu_{n, k}} V_{i}^{2}(k) \geq & \left(\frac{V_{0}}{V}\right)^{\frac{2}{n(n+1)(n-1)^{2}}} \mu_{n, k} \cdot \frac{k+1}{(k !)^{2}}\left(\frac{(n !)^{2}}{n+1}\right)^{\frac{k}{n}} V^{\frac{2 k}{n}} \\
& +\frac{1}{\mu_{n, k}-(n+1-k)} \sum_{1 \leq i<j \leq \mu_{n, k}}\left(V_{i}(k)-V_{j}(k)\right)^{2} .
\end{aligned}
$$

We use the well-known equality $[8,12]$

$$
\sum_{i=1}^{\frac{1}{2} n(n+1)} a_{i}^{2}=(n+1)^{2} R^{2}
$$

and the well-known inequality $[4,13]$

$$
\sum_{i=1}^{\mu_{n, k}} V_{i}^{2}(k) \leq \frac{n !}{n^{k}(k !)^{3}(n-k) !(n+1)^{k-1}}\left(\sum_{i=1}^{\frac{1}{2} n(n+1)} a_{i}^{2}\right)^{k}
$$

Equality holds iff $\Omega_{n}$ is regular. When $k=1$, then (3.13) is the identity.

By (3.12) and (3.13) we get

$$
\sum_{i=1}^{\mu_{n, k}} V_{i}^{2}(k) \leq \frac{(n+1)^{k+1} n !}{(k !)^{3} n^{k}(n-k) !} R^{2 k}
$$

We use the well-known inequality in [8]

$$
V \geq \frac{n^{\frac{n}{2}}(n+1)^{\frac{n+1}{2}}}{n !} r^{n}
$$

with equality iff $\Omega_{n}$ is regular.

From (3.10), (3.14), and (3.15) we can obtain (3.4). By (3.11), (3.14), and (3.15) we can obtain (3.5). It is easy to see that equality holds in (3.4) or (3.5) iff $\Omega_{n}$ is regular.

Let $\omega$ be the width of $\Omega_{n}$. We put

$$
\beta_{n}=\frac{n\left[\frac{n+1}{2}\right]\left(n+1-\left[\frac{n+1}{2}\right]\right)}{(n+1)^{2}} .
$$


In 1977, Alexander [8, 14] proved Sallee's conjecture and obtained the inequality

$$
\omega^{-2} \geq \beta_{n} R^{-2}
$$

Equality holds iff $\Omega_{n}$ is regular.

In 1983, Yang and Zhang [8,15] improved (3.16) and established an inequality as follows:

$$
\omega^{-2} \geq \beta_{n} \frac{(n+1)^{\frac{n+1}{n}}}{n \cdot(n !)^{\frac{2}{n}}} V^{-\frac{2}{n}}
$$

with equality iff $\Omega_{n}$ is regular.

In this section, we shall give generalizations of the equalities (3.16) and (3.17) by using (2.4) and (2.5) in the above section.

Theorem 4 For an $n$-simplex $\Omega_{n}$ and $k=1,2, \ldots, n-1$, we have

$$
\begin{aligned}
& \omega^{-2} \geq\left(\frac{R}{n r}\right)^{\frac{2}{n\left(n^{2}-1\right)}} \beta_{n} \frac{(n+1)^{\frac{n+1}{n}}}{n \cdot(n !)^{\frac{2}{n}}} V^{-\frac{2}{n}}+\beta_{n} \frac{(n+1) V^{-2}}{n^{4}(n-1)} \sum_{0 \leq i<j \leq n}\left(F_{i}-F_{j}\right)^{2}, \\
& \omega^{-2} \geq\left(\frac{V_{0}}{V}\right)^{\frac{2}{n\left(n^{2}-1\right)}} \beta_{n} \frac{(n+1)^{\frac{n+1}{n}}}{n \cdot(n !)^{\frac{2}{n}}} V^{-\frac{2}{n}}+\beta_{n} \frac{(n+1) V^{-2}}{n^{4}(n-1)} \sum_{0 \leq i<j \leq n}\left(F_{i}-F_{j}\right)^{2} .
\end{aligned}
$$

Equality holds iff $\Omega_{n}$ is regular.

Proof We use the well-known inequality in [15] as follows:

$$
\omega \leq\left[\frac{n^{3}(n+1)}{\left[\frac{n+1}{2}\right]\left(n+1-\left[\frac{n+1}{2}\right]\right)}\right]^{\frac{1}{2}} \frac{V}{\left(\sum_{i=0}^{n} F_{i}^{2}\right)^{\frac{1}{2}}} .
$$

Equality holds iff $\Omega_{n}$ is regular.

The above inequality can be written as

$$
\omega^{-2} \geq \beta_{n} \cdot \frac{n+1}{n^{4}} \frac{\sum_{i=0}^{n} F_{i}^{2}}{V^{2}} .
$$

By (3.20) and (2.6) we can get (3.18), and from (3.20) and (2.7) we can get (3.19).

Substituting (2.10) into (3.18) and (3.19) we obtain generalizations of the inequality (3.16) as follows.

Corollary 6 For an n-simplex $\Omega_{n}$ and $k=1,2, \ldots, n-1$, we have

$$
\begin{aligned}
& \omega^{-2} \geq\left(\frac{R}{n r}\right)^{\frac{2}{n\left(n^{2}-1\right)}} \beta_{n} R^{-2}+\beta_{n} \frac{(n+1) V^{-2}}{n^{4}(n-1)} \sum_{0 \leq i<j \leq n}\left(F_{i}-F_{j}\right)^{2}, \\
& \omega^{-2} \geq\left(\frac{V_{0}}{V}\right)^{\frac{2}{n\left(n^{2}-1\right)}} \beta_{n} R^{-2}+\beta_{n} \frac{(n+1) V^{-2}}{n^{4}(n-1)} \sum_{0 \leq i<j \leq n}\left(F_{i}-F_{j}\right)^{2} .
\end{aligned}
$$

Equality holds iff $\Omega_{n}$ is regular. 
For $i=0,1, \ldots, n$, let $h_{i}$ be the altitude of the $i$ th face of the simplex $\Omega_{n}$. The following inequality is well known (see [8]):

$$
V^{2} \geq \frac{n^{n}}{(n !)^{2}(n+1)^{n-1}}\left(\prod_{i=0}^{n} h_{i}\right)^{\frac{2 n}{n+1}}
$$

Equality holds iff $\Omega_{n}$ is regular.

By using the results in Section 2 we can obtain generalizations of the inequality (3.23) as follows.

Theorem 5 For an $n$-simplex $\Omega_{n}$ and $k=1,2, \ldots, n-1$, we have

$$
\begin{aligned}
V^{2\left(n^{2}-1\right) k} \geq & \left(\frac{R}{n r}\right)^{\frac{2 k \alpha}{n(n+1)(n-1)^{2}}} \cdot\left[\frac{n^{n}}{(n !)^{2}(n+1)^{n-1}}\left(\prod_{i=0}^{n} h_{i}\right)^{\frac{2 n}{n+1}}\right]^{\left(n^{2}-1\right) k} \\
& +\frac{(n r)^{2\left(n^{3}-n-1\right) k}}{\mu_{n, k}-(n+1-k)}\left[\frac{(n+1)^{n+1}}{n^{n} \cdot(n !)^{2}}\right]^{\left(n^{2}-1\right) k} \sum_{1 \leq i<j \leq \mu_{n, k}}\left(V_{i}(k)-V_{j}(k)\right)^{2}, \\
V^{2\left(n^{2}-1\right) k} \geq & \left(\frac{V_{0}}{V}\right)^{\frac{2 k \alpha}{n(n+1)(n-1)^{2}}} \cdot\left[\frac{n^{n}}{(n !)^{2}(n+1)^{n-1}}\left(\prod_{i=0}^{n} h_{i}\right)^{\frac{2 n}{n+1}}\right]^{\left(n^{2}-1\right) k} \\
& +\frac{(n r)^{2\left(n^{3}-n-1\right) k}}{\mu_{n, k}-(n+1-k)}\left[\frac{(n+1)^{n+1}}{n^{n} \cdot(n !)^{2}}\right]^{\left(n^{2}-1\right) k} \sum_{1 \leq i<j \leq \mu_{n, k}}\left(V_{i}(k)-V_{j}(k)\right)^{2} .
\end{aligned}
$$

Equality holds iff $\Omega_{n}$ is regular.

Proof We take $k=n-1$ in (2.16) and (2.17), and get

$$
\begin{aligned}
& \prod_{i=0}^{n} F_{i} \geq\left(\frac{R}{n r}\right)^{\frac{1}{n(n-1)}} \frac{n^{\frac{3(n+1)}{2}}}{(n !)^{\frac{n+1}{n}}(n+1)^{\frac{n^{2}-1}{2 n}}} V^{\frac{n^{2}-1}{n}}, \\
& \prod_{i=0}^{n} F_{i} \geq\left(\frac{V_{0}}{V}\right)^{\frac{1}{n(n-1)}} \frac{n^{\frac{3(n+1)}{2}}}{(n !)^{\frac{n+1}{n}}(n+1)^{\frac{n^{2}-1}{2 n}}} V^{\frac{n^{2}-1}{n}} .
\end{aligned}
$$

Using the formula $F_{i}=\frac{n V}{h_{i}}$ (see [8]) and (3.26) we get

$$
V^{2\left(n^{2}-1\right) k} \geq \frac{R^{2 k}}{(n r)^{2 k}}\left[\frac{n^{n}}{(n !)^{2}(n+1)^{n-1}}\left(\prod_{i=0}^{n} h_{i}\right)^{\frac{2 n}{n+1}}\right]^{\left(n^{2}-1\right) k} .
$$

By Theorem 3 we get

$$
R^{2 k} \geq\left(\frac{R}{n r}\right)^{\frac{2 k}{n(n+1)(n-1)^{2}}}(n r)^{2 k}+\frac{1}{\mu_{n, k}-(n+1-k)} \sum_{1 \leq i<j \leq \mu_{n, k}}\left(V_{i}(k)-V_{j}(k)\right)^{2} .
$$


Substituting (3.29) into (3.28) we get

$$
\begin{aligned}
V^{2\left(n^{2}-1\right) k} \geq & \left(\frac{R}{n r}\right)^{\frac{2 k \alpha}{n(n+1)(n-1)^{2}}} \cdot\left[\frac{n^{n}}{(n !)^{2}(n+1)^{n-1}}\left(\prod_{i=0}^{n} h_{i}\right)^{\frac{2 n}{n+1}}\right]^{\left(n^{2}-1\right) k} \\
& +\frac{1}{(n r)^{2 k}\left[\mu_{n, k}-(n+1-k)\right]} \cdot\left[\frac{n^{n}}{(n !)^{2}(n+1)^{n-1}}\left(\prod_{i=0}^{n} h_{i}\right)^{\frac{2 n}{n+1}}\right]^{\left(n^{2}-1\right) k} \\
& \cdot \sum_{1 \leq i<j \leq \mu_{n, k}}\left(V_{i}(k)-V_{j}(k)\right)^{2} .
\end{aligned}
$$

We use the well-known inequality in $[4,8]$ as follows:

$$
\prod_{i=0}^{n} h_{i} \geq(n+1)^{n+1} r^{n+1}
$$

with equality holding iff $\Omega_{n}$ is regular.

From (3.30) and (3.31) we can obtain (3.24). It is easy to see that equality holds iff $\Omega_{n}$ is regular.

By a similar method we can prove that inequality (3.25) is also valid.

Let $P$ be an interior point of $\Omega_{n}$ and $d_{i}$ the distance from the point $P$ to the $i$ th face $f_{i}$ of $\Omega_{n}$ for $i=0,1, \ldots, n$. In [8], Gerber established the following inequality:

$$
\left(\prod_{i=0}^{n} d_{i}\right)^{-2} \geq\left[\frac{n^{n(n+1)}(n+1)^{(n+1)^{2}}}{(n !)^{2(n+1)}}\right]^{\frac{1}{n}} V^{-\frac{2(n+1)}{n}} .
$$

For any natural number $m>0$, Leng and Ma [16] obtained an inequality as follows:

$$
\left[\sum_{0 \leq i<j \leq n}\left(d_{i} d_{i}\right)^{-m}-\left(\mu_{n, 1}\right)^{1-m} \cdot 2 \cdot r^{-2 m}\right]^{\frac{n}{m}} \geq\left[\left(\mu_{n, 1}\right)^{1-m}\left(\left(\mu_{n, 1}\right)^{m}-1\right) n^{2 m} R^{-2 m}\right]^{\frac{n}{m}} .
$$

Equalities hold in (3.32) and (3.33) iff $\Omega_{n}$ is regular and $P$ is the center of $\Omega_{n}$.

By using the results in the Section 2 we can obtain generalizations of (3.32) and (3.33) as follows.

Theorem 6 For an n-simplex $\Omega_{n}$, we have

$$
\begin{aligned}
\left(\prod_{i=0}^{n} d_{i}\right)^{-2} \geq & \left(\frac{R}{n r}\right)^{\frac{2}{n\left(n^{2}-1\right)}} \cdot\left[\frac{n^{n+1}(n+1)^{(n+1)^{2}}}{(n !)^{2(n+1)}}\right]^{\frac{1}{n}} V^{-\frac{2(n+1)}{n}} \\
& +\frac{n^{n-2}(n+1)^{n+2} V^{-4}}{(n !)^{2}(n-1)} \sum_{0 \leq i<j \leq n}\left(F_{i}-F_{j}\right)^{2}, \\
\left(\prod_{i=0}^{n} d_{i}\right)^{-2} \geq & \left(\frac{V_{0}}{V}\right)^{\frac{2}{n\left(n^{2}-1\right)}} \cdot\left[\frac{n^{n+1}(n+1)^{(n+1)^{2}}}{(n !)^{2(n+1)}}\right]^{\frac{1}{n}} V^{-\frac{2(n+1)}{n}} \\
& +\frac{n^{n-2}(n+1)^{n+2} V^{-4}}{(n !)^{2}(n-1)} \sum_{0 \leq i<j \leq n}\left(F_{i}-F_{j}\right)^{2} .
\end{aligned}
$$

Equality holds in (3.32) or (3.33) iff $\Omega_{n}$ is regular and $P$ is the center of $\Omega_{n}$. 
Proof By (2.14), we have

$$
\left(\prod_{i=0}^{n} d_{i}\right)^{-2} \geq \frac{(n+1)^{2(n+1)}}{n^{2(n+1)}} V^{-2(n+1)} \prod_{i=0}^{n} F_{i}^{2} .
$$

We use the well-known inequality in [7] as follows:

$$
\prod_{i=0}^{n} F_{i}^{2} \geq \frac{n^{3 n}}{(n !)^{2}(n+1)^{n}} V^{2(n-1)} \sum_{i=0}^{n} F_{i}^{2}
$$

Equality holds iff $\Omega_{n}$ is regular.

Using (3.37) and (2.6) we get

$$
\begin{aligned}
\prod_{i=0}^{n} F_{i}^{2} \geq & \left(\frac{R}{n r}\right)^{\frac{2}{n\left(n^{2}-1\right)}} \frac{n^{3(n+1)}}{(n !)^{\frac{2(n+1)}{n}}(n+1)^{\frac{n^{2}-1}{n}}} V^{\frac{2\left(n^{2}-1\right)}{n}} \\
& +\frac{n^{3 n} V^{2(n-1)}}{(n !)^{2}(n+1)^{n}(n-1)} \sum_{0 \leq i<j \leq n}\left(F_{i}-F_{j}\right)^{2} .
\end{aligned}
$$

From (3.36) and (3.38) we can obtain (3.34). It is easy to see that equality holds in (3.34) iff $\Omega_{n}$ is regular and $P$ is the center of $\Omega_{n}$.

By a similar method we can prove that (3.35) is valid.

Theorem 7 Let $\Omega_{n}$ be an n-simplex and $m>0$ a natural number, then we have

$$
\begin{aligned}
& {\left[\sum_{0 \leq i<j \leq n}\left(d_{i} d_{j}\right)^{-m}-\left(\mu_{n, 1}\right)^{1-m} r^{-2 m}\right]^{\frac{n}{m}}} \\
& \geq\left(\frac{R}{n r}\right)^{\frac{2}{n(n+1)(n-1)^{2}}}\left[\left(\mu_{n, 1}\right)^{1-m}\left(\left(\mu_{n, 1}\right)^{m}-1\right) n^{2 m} R^{-2 m}\right]^{\frac{n}{m}} \\
& \cdot \gamma(n, m)(R r)^{-2} \sum_{1 \leq i<j \leq \mu_{n, 1}}\left(a_{i}-a_{j}\right)^{2}, \\
& \left.\sum_{0 \leq i<j \leq n}\left(d_{i} d_{j}\right)^{-m}-\left(\mu_{n, 1}\right)^{1-m} r^{-2 m}\right]^{\frac{n}{m}} \\
& \geq\left(\frac{V_{0}}{V}\right)^{\frac{2}{n(n+1)(n-1)^{2}}}\left[\left(\mu_{n, 1}\right)^{1-m}\left(\left(\mu_{n, 1}\right)^{m}-1\right) n^{2 m} R^{-2 m}\right]^{\frac{n}{m}} \\
& \cdot \gamma(n, m)(R r)^{-2} \sum_{1 \leq i<j \leq \mu_{n, 1}}\left(a_{i}-a_{j}\right)^{2} .
\end{aligned}
$$

Equality holds iff $\Omega_{n}$ is regular and $P$ is the center of $\Omega_{n}$, where

$$
\gamma_{n, m}=\left[\left(\mu_{n, 1}\right)^{1-m}\left(\left(\mu_{n, 1}\right)^{m}-1\right)\right]^{\frac{n}{m}} \cdot \frac{2}{n^{2 n+3}(n-1)} .
$$

Proof We use the following well-known inequality (3.8) in [16]:

$$
\sum_{0 \leq i<j \leq n}\left(d_{i} d_{j}\right)^{-m} \geq\left(\mu_{n, 1}\right)^{1-m}\left[r^{-2 m}+(n+1)^{m} n^{m}\left(\frac{n+1}{n !^{2}}\right)^{\frac{n}{m}} \cdot \frac{\left(\mu_{n, 1}\right)^{m}-1}{V^{\frac{2 m}{n}}}\right]
$$


i.e.

$$
\begin{aligned}
& {\left[\sum_{0 \leq i<j \leq n}\left(d_{i} d_{j}\right)^{-m}-\left(\mu_{n, 1}\right)^{1-m} r^{-2 m}\right]} \\
& \quad \geq\left(\mu_{n, 1}\right)^{1-m}(n+1)^{m} n^{m}\left(\frac{n+1}{n !^{2}}\right) \cdot\left[\left(\mu_{n, 1}\right)^{m}-1\right]^{\frac{2 m}{n}} \frac{1}{V^{2}} .
\end{aligned}
$$

Equality holds iff $\Omega_{n}$ is regular and $P$ is the center of $\Omega_{n}$.

From (2.18) we have

$$
\frac{1}{V^{2}} \geq \frac{n !^{2} \cdot n^{n}}{(n+1)^{n+1} R^{2 n}(n r)^{2}} R^{2}
$$

Substituting (3.42) into (3.42) and using (3.6) we can obtain (3.39). It is easy to see that equality holds in (3.39) iff $\Omega_{n}$ is regular and $P$ is the center of $\Omega_{n}$.

The proof of (3.4) is similar.

In fact, the strengthening of some well-known inequalities for simplices can be derived from Theorem 1 and Theorem 2 . In this paper, we omit the details.

\section{Competing interests}

The authors declare that they have no competing interests.

\section{Authors' contributions}

The authors co-authored this paper together. All authors read and approved the final manuscript.

\section{Author details}

${ }^{1}$ School of Mathematics and Statistics, Hefei Normal University, Hefei, Anhui 230601, P.R. China. ${ }^{2}$ Department of Mathematics and Physics, Anhui Xinhua University, Hefei, 230088, P.R. China.

\section{Acknowledgements}

This work is supported by the Doctoral Programs Foundation of Education Ministry of China (20113401110009) and Foundation of Anhui higher school (KJ2013A220); Natural Science Research Project of Hefei Normal University (2012kj11). The authors are grateful for the help.

Received: 6 July 2014 Accepted: 22 January 2015 Published online: 19 February 2015

\section{References}

1. Finsler, P, Hadwiger, H: Inequalities for triangles. Comment. Math. Helv. 10, 316-326 (1937/1938)

2. Bottema, O: Geometric Inequalities. Springer, Berlin (1989)

3. Chen, J, Ma, Y: A class of inequalities involving two simplices. J. Math. Res. Expo. 9(2), 282-284 (1989) (in Chinese)

4. Shen, WX: Introduction on Simplices. Hunan Normal University Press, ChangSha (2000) (in Chinese)

5. Leng, GS, Tang, LH: Some generalizations to several dimensions of the Peode inequality with applications. Acta Math Sin 40(1), 14-21 (1997) (in Chinese)

6. Su, HM: An inequality concerning the volume, edge-lengths and side faces of a simplex. J. Math. 13, 453-454 (1993) (in Chinese)

7. Zhang, JZ, Yang, L: A class of geometric inequalities concerning to mass-point system. J. Univ. Sci. Technol. China 11, 1-8 (1981) (in Chinese)

8. Mitrinović, DS, Pečarić, JE, Volenec, V: Recent Advances in Geometric Inequalities. Kluwer Academic, Dordrecht (1989)

9. Lindensttauss, J, Milman, VD: Geometric Aspects of Functional Analysis. Springer, Berlin (1991)

10. Tóth, LF: Extremum properties of regular polytopes. Acta Math. Acad. Sci. Hung. 6, 143-146 (1995)

11. Kuang, JC: Applied Inequalities. Shandong Science and Technology Press, Jinan (2004) (in Chinese)

12. Yang, SG, Wang, J: Improvement of $n$-dimensional Euler inequality. J. Geom. 51, 190-195 (1995)

13. Yang, L, Zhang, JZ: A class of geometric inequalities for the point system. Acta Math. Sin. 23, 740-749 (1980) (in Chinese)

14. Alexander, R: The width and diameter of a simplex. Geom. Dedic. 6, 87-94 (1977)

15. Yang, L, Zhang, JZ: Metric equation applied to Sallee's conjecture. Acta Math. Sin. 26(4), 488-493 (1983) (in Chinese)

16. Leng, GS, Ma, Y: Inequalities for a simplex and interior point. Geom. Dedic. 85, 1-10 (2001) 